\title{
Integrated epigenetic biomarkers in circulating cell-free DNA as a robust classifier for pancreatic cancer
}

Feng Cao ${ }^{1+}$, Ailin Wei ${ }^{2,3+}$, Xinlei Hu${ }^{1}$, Yijing He ${ }^{1}$, Jun Zhang ${ }^{1}$, Lin Xia ${ }^{1}$, Kailing Tu${ }^{1}$, Jue Yuan², Ziheng Guo ${ }^{2}$, Hongying Liu', Dan Xie ${ }^{1 *}$ and Ang $\mathrm{Li}^{2^{*}}$

\begin{abstract}
Background: The high lethal rate of pancreatic cancer is partly due to a lack of efficient biomarkers for screening and early diagnosis. We attempted to develop effective and noninvasive methods using 5 -methylcytosine $(5 \mathrm{mC})$ and 5-hydroxymethylcytosine $(5 \mathrm{hmC})$ markers from circulating cell-free DNA (cfDNA) for the detection of pancreatic ductal adenocarcinoma (PDAC).

Results: A 24-feature $5 \mathrm{mC}$ model that can accurately discriminate PDAC from healthy controls (area under the curve $(A \cup C)=0.977$, sensitivity $=0.824$, specificity $=1$ ) and a $5 \mathrm{hmC}$ prediction model with 27 features demonstrated excellent detection power in two distinct validation sets (AUC $=0.992$ and 0.960 , sensitivity $=0.786$ and 0.857 , specificity $=1$ and 0.993 ). The 51 -feature model combining $5 \mathrm{mC}$ and $5 \mathrm{hmC}$ markers outperformed both of the individual models, with an AUC of 0.997 (sensitivity $=0.938$, specificity $=0.955$ ) and particularly an improvement in the prediction sensitivity of PDAC. In addition, the weighted diagnosis score (wd-score) calculated with the $5 \mathrm{hmC}$ model can distinguish stage I patients from stage II-IV patients.
\end{abstract}

Conclusions: Both $5 \mathrm{mC}$ and $5 \mathrm{hmC}$ biomarkers in cfDNA are effective in PDAC detection, and the $5 \mathrm{mC}-5 \mathrm{hmC}$ integrated model significantly improve the detection sensitivity.

Keywords: Cell-free $5 \mathrm{mC}$ sequencing, Cell-free $5 \mathrm{hmC}$ sequencing, Liquid biopsy, Pancreatic cancer, Cancer diagnosis

\section{Background}

Pancreatic cancer is a highly lethal disease, as most patients are asymptomatic until they are in an advanced stage [1]. Pancreatic ductal adenocarcinoma (PDAC) patients benefit most from early diagnosis and surgery. Once distant metastasis occurs, any systemic therapy is

\footnotetext{
* Correspondence: danxie@scu.edu.cn; angli@scu.edu.cn

${ }^{\dagger}$ Feng Cao and Ailin Wei contributed equally to this work.

${ }^{1}$ Frontier Science Center for Disease Molecular Network, State Key Laboratory of Biotherapy, West China Hospital, Sichuan University, Chengdu 610041, Sichuan Province, China

${ }^{2}$ Department of Pancreatic Surgery, West China Hospital, Sichuan University, Chengdu 610041, Sichuan Province, China

Full list of author information is available at the end of the article
}

rarely curative. Therefore, a major goal in PDAC research is the detection of cancer when effective surgery can be performed.

Liquid biopsies, a well-known noninvasive method, have aroused public attention as diagnostic materials for cancer, particularly circulating tumor DNA (ctDNA) in plasma. Taking advantage of technical advances, both genetic and epigenetic aberrations of cell-free DNA (cfDNA) can be detected [2] and have shown promising performance in clinical practice, including diagnosis [38], prognosis [9-12], and drug resistance [13, 14].

Epigenetic mechanisms play critical roles in individual development and tissue-specific gene expression, while

(c) The Author(s). 2020 Open Access This article is licensed under a Creative Commons Attribution 4.0 International License, which permits use, sharing, adaptation, distribution and reproduction in any medium or format, as long as you give appropriate credit to the original author(s) and the source, provide a link to the Creative Commons licence, and indicate if changes were made. The images or other third party material in this article are included in the article's Creative Commons licence, unless indicated otherwise in a credit line to the material. If material is not included in the article's Creative Commons licence and your intended use is not permitted by statutory regulation or exceeds the permitted use, you will need to obtain permission directly from the copyright holder. To view a copy of this licence, visit http://creativecommons.org/licenses/by/4.0/ The Creative Commons Public Domain Dedication waiver (http://creativecommons.org/publicdomain/zero/1.0/) applies to the data made available in this article, unless otherwise stated in a credit line to the data. 
their dysregulation frequently occurs in human diseases, notably cancer $[15,16]$. Global changes in epigenetic modifications, such as 5 -methylcytosine $(5 \mathrm{mC})$ and 5-hydroxymethylcytosine $(5 \mathrm{hmC})$, are hallmarks of cancer [17, 18]. It was suggested that epigenetic modifiers, which are regulated by epigenetic modulators serving to transduce signals from environmental factors, modified tumor progenitor genes to influence gene expression, which was believed to be the earliest stage of carcinogenesis [19].

Considering the low mutation frequency of tumorrelated somatic mutations and the limited detection sensitivity, $5 \mathrm{mC}$ and $5 \mathrm{hmC}$ in cfDNA could serve as parallel or even more valuable biomarkers [20]. Recent technological improvements in $5 \mathrm{mC}$ and $5 \mathrm{hmC}$ detection from cfDNA, including the cell-free methylated DNA immunoprecipitation and highthroughput sequencing (cfMeDIP-seq) method [21, 22 ] and cell-free $5 \mathrm{hmC}$ sequencing methods reported recently [20, 23], offer substantial advantages over previous ctDNA $5 \mathrm{mC}$ and $5 \mathrm{hmC}$ detection methods. Therefore, $5 \mathrm{mC}$ detection and $5 \mathrm{hmC}$ characterization in cfDNA are anticipated to be robust and costeffective methods for clinical application in cancer diagnosis and therapy [24].

We employed cfMeDIP-seq and cell-free $5 \mathrm{hmC}$ sequencing to explore the application potential of epigenetic markers in noninvasive diagnosis and attempted to test whether the combination of the two types of epigenetic markers could improve the diagnostic power. We developed three prediction models for PDAC detection, including a $5 \mathrm{mC}$ model with 24 features, a $5 \mathrm{hmC}$ model with 27 features, and an integrated model using the 51 features identified. In addition, we investigated the genomic distribution of $5 \mathrm{mC}$ and $5 \mathrm{hmC}$ as well as the modification level changes at H3K36me3, H3K27ac, H3K4me3, H3K4me1, and H3K27me3.

\section{Results}

\section{Characterization of the cfMeDIP-seq data and cfDNA $5 \mathrm{hmC}$ sequencing data}

We recruited 136 healthy individuals and 72 PDAC patients of Chinese descent in our study (Table 1), of which 61 PDAC and 86 healthy controls had paired cellfree $5 \mathrm{mC}$ and $5 \mathrm{hmC}$ data. CfMeDIP-seq data from 97 healthy controls and 67 PDAC samples were qualified for methylome analysis. The spike-in control with sequencing adaptors demonstrated specific $5 \mathrm{mC}$ enrichment (Supplementary Figure 1A). The median final unique non-duplicate mapping rate of the cfMeDIP-seq libraries was 0.8 , and the median total reads was $\sim 17.4$ M (Supplementary Table 1).
Considering that cfDNA $5 \mathrm{hmC}$ signatures in PDAC also deserve deep inquiry, $5 \mathrm{hmC}$ profiling data from 136 healthy controls and 67 PDAC samples were generated. The count of reads mapping to the spike-in control demonstrated highly specific enrichment of $5 \mathrm{hmC}$ fragments (Supplementary Figure 1B). The final $5 \mathrm{hmC}$ libraries were highly complex (a median unique nonduplicate rate of 0.83 ) with a relatively low sequencing depth (median 20.8 M reads) (Supplementary Table 2).

\section{Genome-wide profiling of $5 \mathrm{mC}$ and $5 \mathrm{hmC}$ in cfDNA}

To explore the distribution patterns of methylation in cfDNA across the genome, we defined the $201 \mathrm{bp}$ fixedwidth peaks called by MACS2 as $5 \mathrm{mC}$-enriched regions. Comparing the peak number between PDAC samples and healthy controls, no significant difference was observed, though the median peak number of PDAC was greater than that of the control group (Supplementary Figure 2A). However, the total number of $5 \mathrm{hmC}$ peaks captured from the PDAC samples was significantly less than that captured from the healthy controls $(P$ value $=$ 1.43E-05) (Supplementary Figure 2B). Considering that the peak number could not fully represent the global modification level, we inspected the $5 \mathrm{mC}$ change with the Integrative Genomics Viewer (IGV) [25, 26]. No significant global $5 \mathrm{mC}$ depletion was observed in the PDAC samples, only demethylation within relatively small ranges. In contrast, global hypermethylation regions were observed (Supplementary Figure 2C). Next, we checked the global $5 \mathrm{hmC}$ level change by IGV. The result showed global $5 \mathrm{hmC}$ loss in the PDAC samples (Supplementary Figure 2D).

By locating these peaks to distinct genome elements, we observed that the $5 \mathrm{mC}$-enriched regions were significantly enriched in CDS, exons, 5'UTRs, 3'UTRs, and promoters, with no significant change in the PDAC samples compared to the controls, while depleted in introns and intergenic regions (Supplementary Figure 3A, B).

Analysis of the $5 \mathrm{hmC}$ peaks showed that the $5 \mathrm{hmC}$ enriched regions are highly enriched in CDS, 5'UTRs, exons, 3'UTRs, and promoters, while depleted in introns and intergenic regions. The PDAC samples showed a significantly higher odds ratio in 5'UTRs, 3'UTRs, and exons, while no significant difference was observed within promoters and CDS compared to the controls (Supplementary Figure 3C, D).

Comparing the distributions of the $5 \mathrm{mC}$ and $5 \mathrm{hmC}$ peaks First, we compared the peak numbers of the $5 \mathrm{mC}$ and $5 \mathrm{hmC}$ profiles. The total number of $5 \mathrm{mC}$ peaks $(n=772$, $365)$ was approximately 2 -folds greater than that of the $5 \mathrm{hmC}$ peaks (Fig. 1a). However, only $16.7 \%$ overlapped with $5 \mathrm{hmC}$ peak sites. Moreover, $30 \%$ of $5 \mathrm{hmC}$ sites also had $5 \mathrm{mC}$ modifications (Supplementary Figure 4A). There 
Table 1 Demographical and clinicopathological characteristics of all the participants

\begin{tabular}{|c|c|c|c|}
\hline Parameters & PDAC group $(N=72)$ & Healthy control group $(N=136)$ & $P$ value \\
\hline Age, average \pm standard error & $59.54 \pm 1.19$ & $58.23 \pm 0.75$ & 0.379 \\
\hline \multicolumn{4}{|l|}{ Gender, $n(\%)$} \\
\hline Male & $41(57 \%)$ & $48(35 \%)$ & 0.003 \\
\hline $\mathrm{BMI}$, average \pm standard error & $22.03 \pm 0.37$ & $23.81 \pm 0.30$ & $<0.0001$ \\
\hline Smoking history, $n(\%)$ & $22(31 \%)$ & 19(14\%) & 0.004 \\
\hline Alcohol history, $n(\%)$ & $25(35 \%)$ & 19(14\%) & $<0.0001$ \\
\hline \multicolumn{4}{|l|}{ Chronic disease, $n(\%)$} \\
\hline Hypertension & $9(13 \%)$ & $37(27 \%)$ & 0.015 \\
\hline Type II diabetes & $13(18 \%)$ & $15(11 \%)$ & 0.158 \\
\hline CA199, average \pm standard error & $357.29 \pm 42.28$ & / & \\
\hline Jaundice & $21(29 \%)$ & 0 & $<0.0001$ \\
\hline Tumor size, average \pm standard error & $3.79 \pm 0.19$ & / & \\
\hline Primary cancer site, $n$ (\%) & & / & \\
\hline Uncinate process & 13(18\%) & & \\
\hline Head & $29(40 \%)$ & & \\
\hline Body & $17(24 \%)$ & & \\
\hline Tail & $13(18 \%)$ & & \\
\hline Surgery, $n(\%)$ & & / & \\
\hline Pancreaticoduodenectomy & $26(36 \%)$ & & \\
\hline Distal pancreatectomy & $11(15 \%)$ & & \\
\hline Palliative intervention techniques & $35(49 \%)$ & & \\
\hline AJCC staging, $n(\%)$ & & / & \\
\hline । & $8(11 \%)$ & & \\
\hline$\|$ & 28(39\%) & & \\
\hline III & $18(25 \%)$ & & \\
\hline IV & $18(25 \%)$ & & \\
\hline
\end{tabular}

BMI body mass index, CA199 carbohydrate antigen199, AJCC American Joint Committee on Cancer

Note: $P$ values were calculated using chi-squared test

were 17,340 genes carrying both $5 \mathrm{mC}$ and $5 \mathrm{hmC}$ modifications, of which 3303 genes ( $16 \%$ of the total genes detected with $5 \mathrm{mC}$ and/or $5 \mathrm{hmC}$ modification) had $5 \mathrm{mC}$ modifications only, and 124 genes were specifically modified with $5 \mathrm{hmC}$ (Supplementary Figure 4B). The results suggested that over $80 \%$ of $5 \mathrm{mC}$ peaks occurred at sites distinct to $5 \mathrm{hmC}$, while genes frequently bear the two types of modifications.

Next, we investigated the functions of the genes with specific $5 \mathrm{mC}$ or specific $5 \mathrm{hmC}$ modifications. The $5 \mathrm{mC}$ specific genes were mainly enriched in the olfactory signaling pathway, keratinization, and GPCR ligand binding, et al. (Supplementary Figure $5 \mathrm{~A}$ ), while the $5 \mathrm{hmC}$ specific genes were enriched in oxidative stress-induced senescence (Supplementary Figure 5B). Supplementary Figure 6 demonstrates examples of $5 \mathrm{mC}$-specific genes and $5 \mathrm{hmC}$-specific genes.

Comparing the genome distribution patterns of $5 \mathrm{hmC}$ and $5 \mathrm{mC}$, we observed that $5 \mathrm{hmC}$ exhibited significantly higher enrichment in CDS, 5'UTRs, 3' UTRs, exons, and promoters. Both $5 \mathrm{mC}$ and $5 \mathrm{hmC}$ demonstrated depletion in introns and intergenic regions. $5 \mathrm{hmC}$ depletion within intergenic regions in PDAC samples showed a much greater extent than $5 \mathrm{mC}$, while the opposite trend was observed within introns (Fig. 1b). Reduced $5 \mathrm{hmC}$ modifications within gene bodies in PDAC were observed by metagene analysis (Fig. 1c). The changes of $5 \mathrm{mC}$ modifications within gene bodies were not as significant as that of the $5 \mathrm{hmC}$ modifications in the regions in the PDAC samples (Fig. 1c).

Given that histone modifications have a biological relationship with DNA methylation [27], we investigated the overlap of $5 \mathrm{mC}$ and $5 \mathrm{hmC}$ modification peaks in five types of histone modifications, including H3K36me3, H3K27ac, H3K4me1, H3K4me3, and H3K27me3. The intersection of $5 \mathrm{hmC}$ profiling data with the histone map of the PANC-1 cell line from the ENCODE Project 

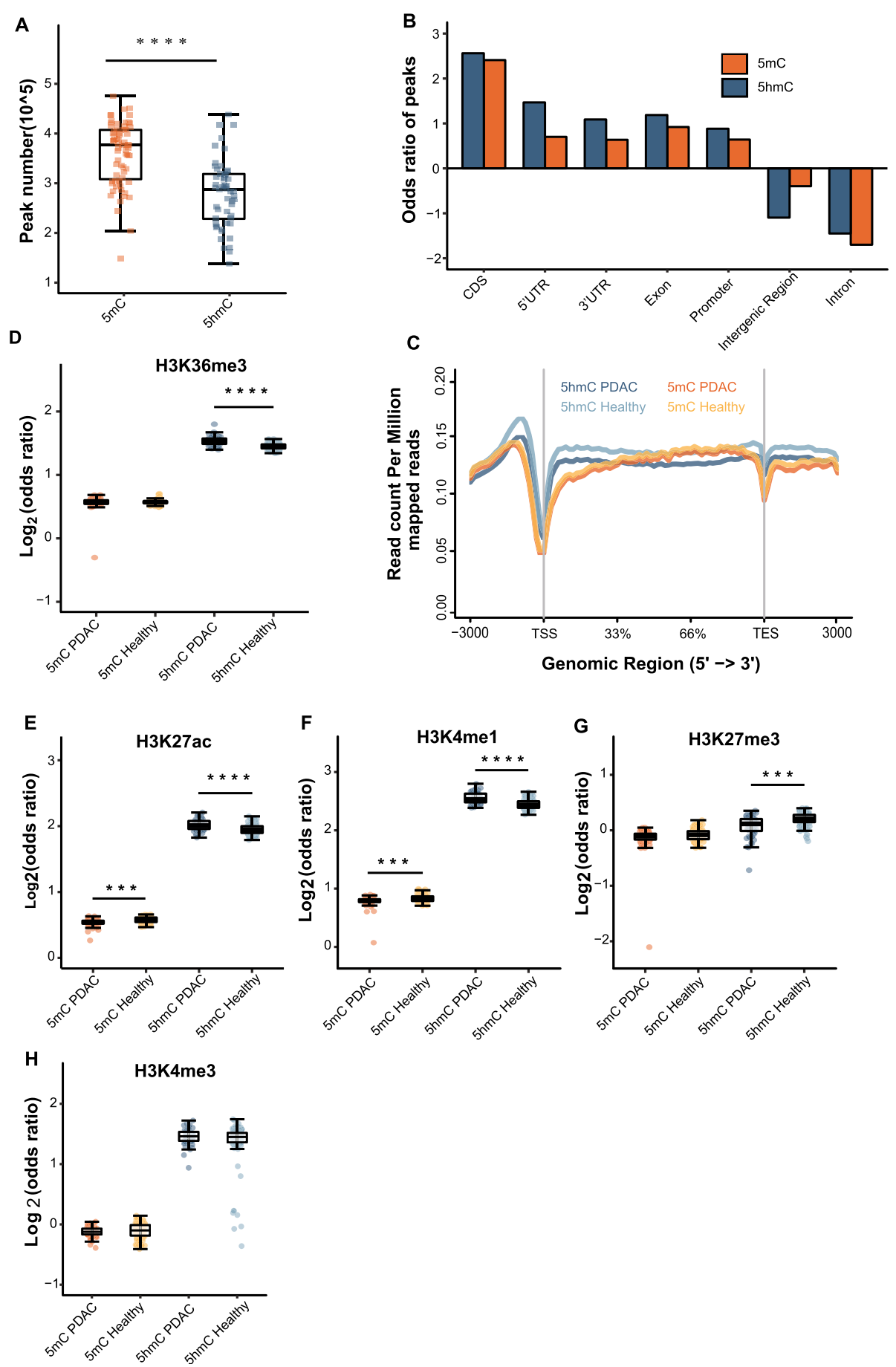

Fig. 1 Comparison of genome-wide distribution between $5 \mathrm{mC}$ and $5 \mathrm{hmC}$. a Boxplot of the total number of the $5 \mathrm{mC}$ peaks and $5 \mathrm{hmC}$ peaks in PDAC samples. $\mathbf{b}$ Enrichment analysis of the $5 \mathrm{mC}$ and $5 \mathrm{hmC}$ peaks overlapping with distinct genomic elements. c Metagene profiles of the regions from TSS to TES with the flanking $3 \mathrm{~kb}$. Boxplots of $\mathrm{Log}_{2}$ (odds ratio) of peaks overlapping with H3K36me3 (d), H3K27ac (e), H3K4me1 (f), H3K27me3 (g), and H3K4me3 (h). ${ }^{*} P<0.05,{ }^{* *} P<0.01,{ }^{* * *} P<0.001$, ${ }^{* * *} P<1 \mathrm{e}-5$, Wilcoxon test. PDAC, pancreatic ductal adenocarcinoma; TSS, transcription start sites; TES, transcription end sites

exhibited increased fragments per kilobase of gene per million mapped reads (FPKM) in H3K36me3 $(P$ value $=$ 7.25E-12), H3K27ac $(P$ value $=2.55 \mathrm{E}-06)$, and H3K4me1 $(P$ value $=1.79 \mathrm{E}-09)$ in the PDAC cohorts relative to the healthy cohorts (Fig. 1d-f). In contrast, $5 \mathrm{mC}$ modifications located in $\mathrm{H} 3 \mathrm{~K} 4 \mathrm{me1}(P$ value $=$ $2.73 \mathrm{E}-05)$ and $\mathrm{H} 3 \mathrm{~K} 27 \mathrm{ac}(P$ value $=1.59 \mathrm{E}-05)$ peaks decreased in the PDAC samples compared to those in the 
healthy control samples (Fig. 1d, e). Given that H3K36me3, H3K27ac, and H3K4me1 are marks of active regulatory elements [28], increased $5 \mathrm{hmC}$ levels and reduced $5 \mathrm{mC}$ levels in these regions suggested the active transcription of genes regulated by the elements. For the repressive histone modification H3K27me3, $5 \mathrm{hmC}$ level significantly decreased in PDAC samples $(P$ value $=5.80 \mathrm{E}-05)$, while $5 \mathrm{mC}$ level changes were not statistically significant (Fig. 1g). The density of both $5 \mathrm{hmC}$ and $5 \mathrm{mC}$ in $\mathrm{H} 3 \mathrm{~K} 4 \mathrm{me} 3$ sites presented no significant difference (Fig. 1h).

\section{Prediction of PDAC by using $5 \mathrm{mC}$ biomarkers in cfDNA}

The fragments per kilobase of gene per million mapped reads (FPKM) of each peak were calculated in every single sample regarding its $5 \mathrm{mC}$ modification level. T-distributed stochastic neighbor embedding ( $t$ SNE) analysis with the consensus peak sets showed a slight batch effect, which was eliminated by Combat (sva package) (Supplementary Figure 7). Based on the consensus peak sets and datasets without batch effects, we identified 688 differentially methylated peaks (DMPs) in the 67 PDAC samples and 97 healthy controls analyzed (Student's $t$ test, $P$ value $<0.01, \mid \log$ fold change $(\mathrm{FC}) \mid>0.8)$, including 560 hypermethylated peaks in the cases and 128 hypermethylated peaks in the controls (Supplementary Table 3). The healthy controls and PDAC samples could be classified by hierarchical clustering and $t$-SNE using the 688 DMPs identified, and no association with resectable stage and jaundice was observed (Fig. 2a, b). Utilizing the above methods and criteria to identify DMPs, the elastic-net method was used for model construction with the set of 164 samples, partitioned randomly into a training set consisting of 50 PDAC samples and 67 healthy controls and a validation set comprising 17 PDAC samples and 30 healthy controls (Supplementary Figure 8). Finally, 24 biomarkers $($ alpha $=0.2) \quad($ Supplementary Table 4$)$ that appeared in at least 3 training subsets were selected for the final model, achieving a sensitivity of $100 \%$ and a specificity of $100 \%$ (area under the curve $($ AUC) $=1$ ) (Fig. 2c) in the training set. To assess the prediction ability of the classifier, 17 PDAC samples and 30 healthy controls were used for validation, exhibiting a sensitivity of $82.4 \%$ and a specificity of $100 \%$ (AUC = 0.971) (Fig. 2c).

The differences in the weighted diagnosis scores (wd-scores) calculated with the model (Supplementary Table 5) between the PDAC group and the healthy group were statistically significant $(P$ value $=1.15 \mathrm{E}$ -22) (Fig. 2d). Unexpectedly, the wd-score divergence between PDAC patients with jaundice and patients without jaundice was statistically significant $(P$ value
$=4.64 \mathrm{E}-03)$ (Fig. 2e). However, no significant difference in the wd-score was observed between the resectable PDAC group and unresectable group, despite the former obtaining a higher median wd-score (Fig. 2f). A portion of the markers identified in this model were mapped to tumor-related genes, such as $M C U$, KRT80, and MGAT5. Figure $2 \mathrm{~g}$ exhibited the IGV plot of the marker locating in $M C U$ gene. The PDAC samples showed increased $5 \mathrm{mC}$ level of the marker when compared with the healthy controls.

\section{Prediction of PDAC by using $5 \mathrm{hmC}$ biomarkers in cfDNA}

No significant batch effects were observed among all cfDNA $5 \mathrm{hmC}$ sequencing data (Supplementary Figure 9). We performed Student's $t$ test to determine the optimal parameter for differentially hydroxymethylated peak (DhMP) identification, and 15 DhMP features were characterized within 53 PDAC samples and 106 healthy controls (Student's $t$ test, $P$ value $<0.001$, $|\log 2 \mathrm{FC}|>=0.8)$, comprising 6 hyperhydroxymethylated peaks and 9 hypohydroxymethylated peaks in the cases (Supplementary Table 6). T-SNE analysis and hierarchical clustering of 53 PDAC samples and 106 healthy control samples with the 15 DhMPs can separate PDAC from healthy controls (Fig. 3a, b). To explore the potential diagnostic value of $5 \mathrm{hmC}$ cfDNA and search for more effective biomarkers, the 159 total samples were randomly divided into a training set and a validation set comprising $75 \%$ and $25 \%$ of the data, respectively, with the elastic-net regularized regression method employed (Supplementary Figure 10). This approach suggested a set of 27 features of $5 \mathrm{hmC}$ profiles (Supplementary Table 7), resulting a discrimination model demonstrating high accuracy in both the training set (sensitivity $=97.4 \%$, specificity $=$ $100 \%$, AUC $=1$ ) and the validation set (sensitivity = $78.6 \%$, specificity $=100 \%$, and AUC $=0.992$ ) (Fig. 3c).

Furthermore, 14 PDAC samples and 30 healthy controls were used to validate the model's performance, achieving a sensitivity of $85.7 \%$ and a specificity of $93.3 \%$ $($ AUC $=0.960)($ Fig. 3d). The wd-score showed an upward trend from healthy controls to PDAC patients, with significantly higher wd-scores in the PDAC samples $(P$ value $=5.98 \mathrm{E}-30)($ Fig. $3 \mathrm{e})$. Interestingly, the wdscore between early-stage (American Joint Committee on Cancer (AJCC) stage I) and late-stage (AJCC stages II, III, and IV) patients demonstrated statistically significant disparity ( $P$ value $=7.00 \mathrm{E}-03)$, which suggested the capacity of the model to discriminate early- from latestage patients (Fig. 3f). No significant difference between the resectable and unresectable PDAC groups was observed (Supplementary Figure 11A), while a significant difference in the wd-score was observed between the groups with and without jaundice (4.60E-02) 

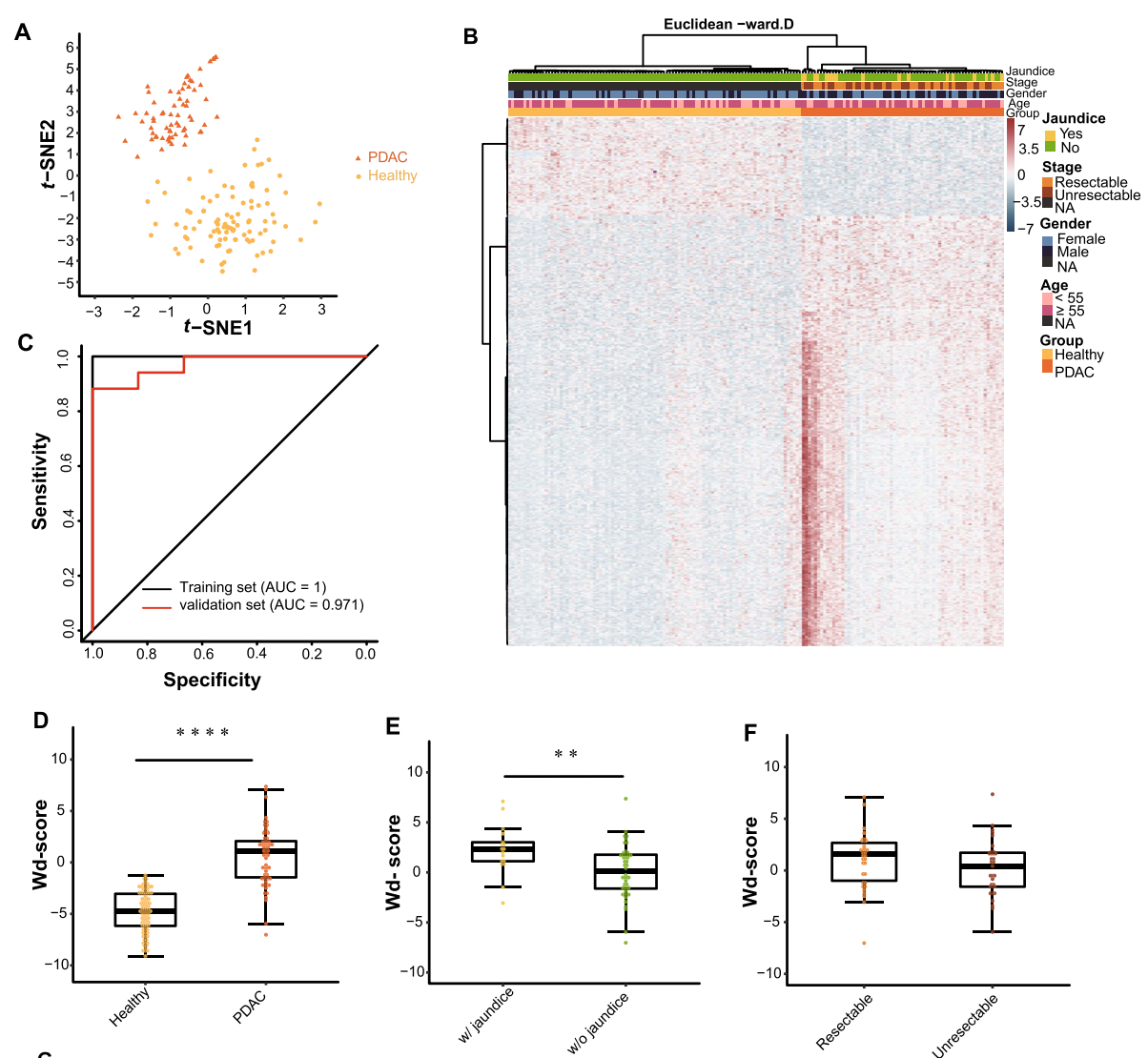

G

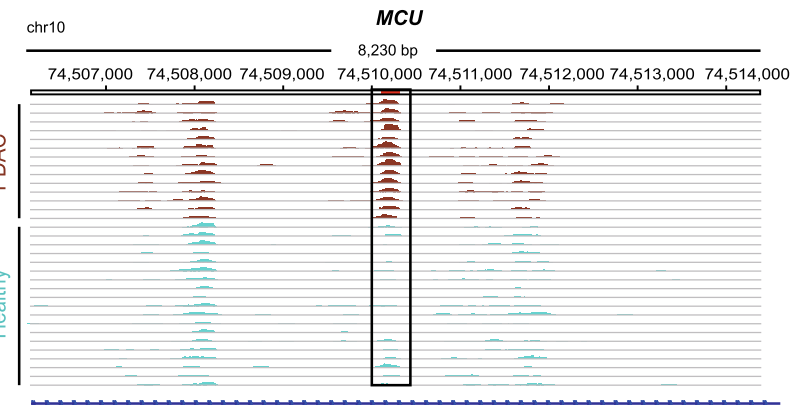

Fig. 2 Cell-free $5 \mathrm{mC}$ for detection of PDAC. T-SNE plot (a) and heatmap (b) of $5 \mathrm{mC}$ FPKM from the training set and the validation set based on the 688 differentially methylated peaks (DMPs). Hierarchical clustering was performed across peaks and samples. $\mathbf{c}$ Performance of the $5 \mathrm{mC}$ model in the training set and the validation set. $\mathbf{d}$ Boxplot of the wd-scores calculating with the $5 \mathrm{mC}$ model for the PDAC samples and the healthy controls. e Boxplot of the wd-scores of the PDAC patients with jaundice and those without jaundice. $\mathbf{f}$ Boxplot of the wd-scores of the resectable PDAC patients and the unresectable patients. $\mathbf{g}$ Genome Browser view of the $5 \mathrm{hmC}$ peaks in MCU gene in chromosome 10 shows a marker locating within the gene (boxed region: chr10: 74510104-74510305). ${ }^{*} P<0.05$, ${ }^{* *} P<0.01,{ }^{* * *} P<0.001$, ${ }^{* * *} P<1 \mathrm{e}-5$, Wilcoxon test. PDAC, pancreatic ductal adenocarcinoma; AUC, area under the curve; wd-score, weighted diagnosis score

(Supplementary Figure 11B). Figure $3 \mathrm{~g}$ presents the $5 \mathrm{hmC}$ biomarker located at the RABGAP1L gene, which plays a key role in tumorigenesis. The $5 \mathrm{hmC}$ modification level of the DhMP at the RABGAP1L gene reduced in PDAC patients versus normal controls.

\section{Integrated model of $5 \mathrm{mC}-5 \mathrm{hmC}$ biomarkers}

Because $5 \mathrm{mC}$ and $5 \mathrm{hmC}$ depict different aspects of the epigenome, we envisioned that conjoint analysis would increase diagnostic power. To test this hypothesis, the $5 \mathrm{hmC}$ features $(n=27)$ and $5 \mathrm{mC}$ features $(n=24)$ identified were combined to construct a classification model with paired $5 \mathrm{mC}$ and $5 \mathrm{hmC}$ datasets from PDAC samples $(n=61)$ and healthy samples $(n=86)$. Both hierarchical clustering and $t$-SNE analysis indicated that the 51 features can discriminate PDAC from healthy samples (Fig. 4a, b). With the elastic-net modeling method, sensitivities of $97.8 \%$ and $93.8 \%$ and specificities of $100 \%$ 

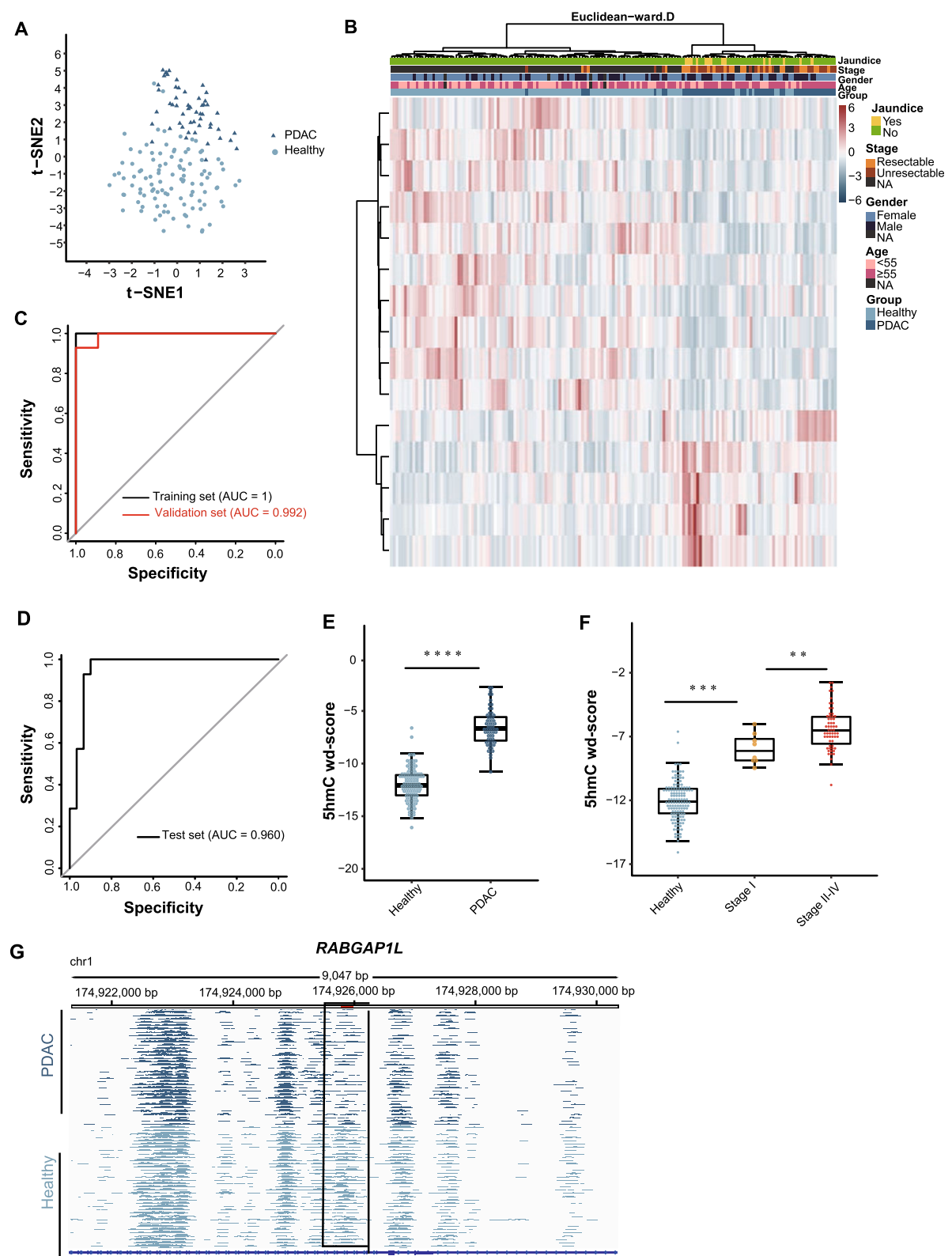

Fig. 3 Cell-free 5hmC for detection of PDAC. T-SNE plot (a) and heatmap (b) of 5hmC FPKM from the training set and the validation set based on the 15 differentially hydroxymethylated peaks (DhMPs). Hierarchical clustering was performed across peaks and samples. c Performance of the $5 \mathrm{hmC}$ model in the training set and the validation set. $\mathbf{d}$ Performance of the $5 \mathrm{hmC}$ model in the test data set. e Boxplot of the wd-scores calculating with $5 \mathrm{hmC}$ model for PDAC samples and healthy controls. $\mathbf{f}$ Boxplot of the wd-scores calculating with $5 \mathrm{hmC}$ model for healthy controls, stage I PDAC samples and stage II-IV PDAC samples. ${ }^{*} P<0.05$, ${ }^{* *} P<0.01$, ${ }^{* *} P<0.001$, ${ }^{* * *} P<1 \mathrm{e}-5$, ANOVA. g Genome Browser view of the $5 \mathrm{hmC}$ peaks in RABGAP1L gene in chromosome 6 shows a marker locating within the gene (boxed region: chr1: 174799728-174799929). PDAC, pancreatic ductal adenocarcinoma; AUC, area under the curve; wd-score, weighted diagnosis score

and $95.5 \%$ (AUC $=0.999$ and 0.997) (Fig. 4c) were achieved in the training and validation datasets respectively, exhibiting better performance than the models using the $5 \mathrm{hmC}$ or $5 \mathrm{mC}$ biomarkers alone. We also applied this model to calculate the wd-score for every single sample. The disparity of the wd-score between PDAC and healthy controls obtained from the integrated model was statistically significant ( $P$ value $=8.45 \mathrm{E}-25)($ Fig. $4 \mathrm{~d})$, as well as the wd-score difference between PDAC patients with jaundice and those without jaundice $(P$ value $=6.28 \mathrm{E}-03)($ Fig. $4 \mathrm{e})$. However, the wd-score of the integrated model failed to classify the resectable and unresectable PDAC samples (Fig. 4f). 

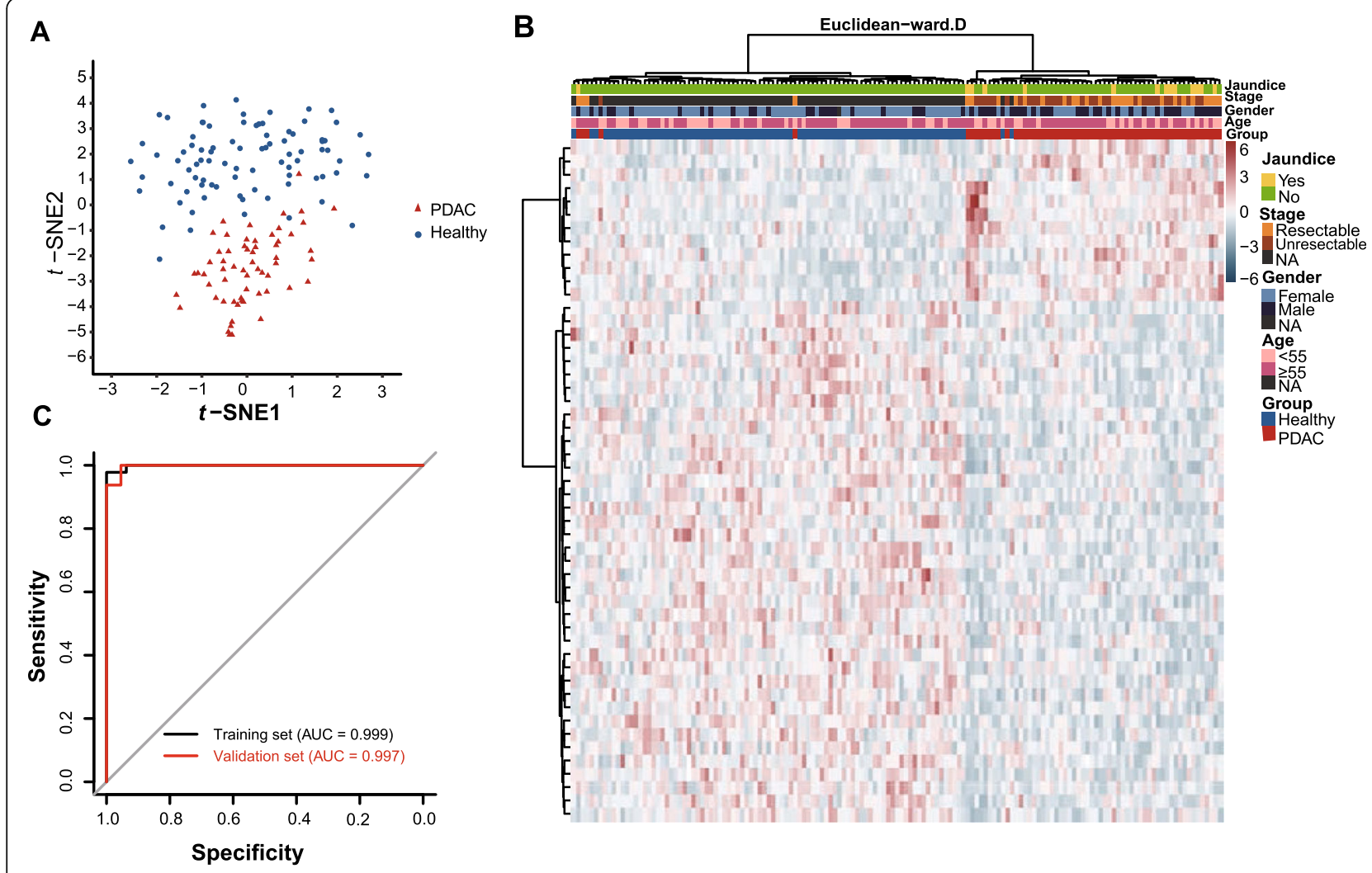

D

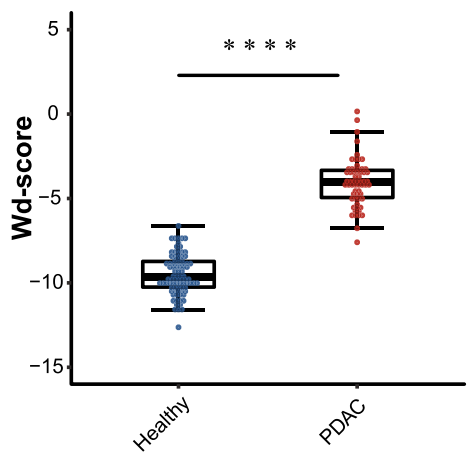

$\mathbf{E}$

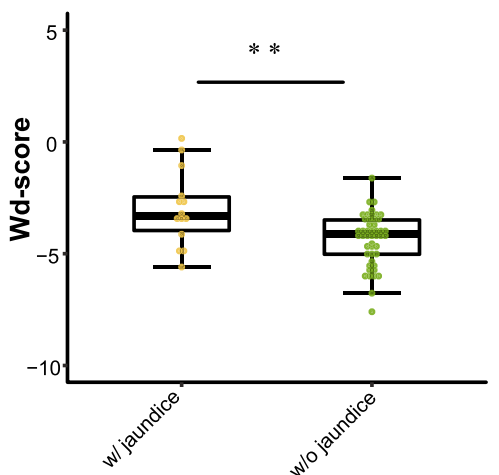

$\mathbf{F}$

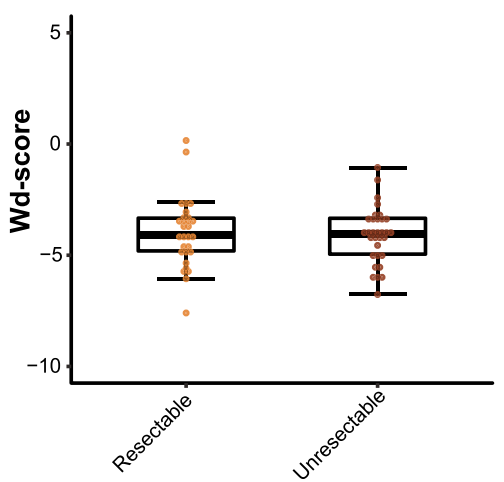

Fig. 4 Performance of the $5 \mathrm{mC}-5 \mathrm{hmC}$ integrated model for PDAC detection. T-SNE plot (a) and heatmap (b) of FPKM from the paired $5 \mathrm{mC}$ and $5 \mathrm{hmc}$ data, based on the 51 features comprising the twenty-four $5 \mathrm{mC}$ biomarkers and the twenty-seven $5 \mathrm{hmC}$ biomarkers. Hierarchical clustering was performed across peaks and samples. c Performance of the $5 \mathrm{mC}-5 \mathrm{hmC}$ integrated model in the training set and the validation set. $\mathbf{d}$ Boxplot of wd-score deriving from the integrated model for the PDAC samples and the healthy controls. e Boxplot of the wd-scores from the integrated model for PDAC patients with jaundice and those without jaundice. $\mathbf{f}$ Boxplot of the wd-scores from the integrated model for the resectable PDAC patients and the unresectable PDAC patients. ${ }^{*} P<0.05,{ }^{* *} P<0.01,{ }^{* * *} P<0.001,{ }^{* * *} P<1 \mathrm{e}-5$, Wilcoxon test. PDAC, pancreatic ductal adenocarcinoma; AUC, area under the curve; wd-score, weighted diagnosis score

Patients with tumor size less than $3 \mathrm{~cm}(P$ value $=1.9 \mathrm{E}$ -02) had significantly higher wd-scores (Table 2). Notably, compared with non-nerve invasive resectable PDAC patients $(n=29)$, patients with nerve invasion had significant higher wd-scores $(P$ value $=2.7 \mathrm{E}-2)$. No significant differences in vascular invasion or positive lymph node metastasis were found in resectable PDAC patients.
In summary, the integrated prediction model demonstrated higher prediction sensitivity ( 10\% higher), notably in stage I samples (with a sensitivity of $87.5 \%$ in the integrated model, and $75.0 \%$ and $62.5 \%$ in the $5 \mathrm{mC}$ and $5 \mathrm{hmC}$ model, respectively) (Supplementary Table 5), supporting the prospect of applying the combined cell-free $5 \mathrm{mC}$ and $5 \mathrm{hmC}$ biomarkers for a more accurate cancer diagnosis. 
Table 2 Comparison of wd-scores (the integrated model) of risk factors and prognosis associated with pancreatic cancer $(n=61)$

\begin{tabular}{|c|c|c|c|}
\hline Variable & Variable level & Wd-score & $P$ value \\
\hline \multirow[t]{2}{*}{$\overline{\text { Age }}$} & $\geq 60$ & $-3.894 \pm 0.257$ & 0.383 \\
\hline & $<60$ & $-4.2126 \pm 0254$ & \\
\hline \multirow[t]{2}{*}{ Gender } & Male & $-3.895 \pm 0.230$ & 0.355 \\
\hline & Female & $-4.235 \pm 0.289$ & \\
\hline \multirow[t]{2}{*}{ Smoking history } & Yes & $-4.079 \pm 0.375$ & 0.911 \\
\hline & No & $-4.032 \pm 0.206$ & \\
\hline \multirow[t]{2}{*}{ Alcohol history } & Yes & $-4.100 \pm 0.314$ & 0.831 \\
\hline & No & $-4.017 \pm 0.224$ & \\
\hline \multicolumn{4}{|l|}{ Chronic disease } \\
\hline \multirow[t]{2}{*}{ Hypertension } & Yes & $-4.094 \pm 0.372$ & 0.911 \\
\hline & No & $-4.038 \pm 0.201$ & \\
\hline \multirow[t]{2}{*}{ Type II diabetes } & Yes & $-3.622 \pm 0.235$ & 0.118 \\
\hline & No & $-4.139 \pm 0.213$ & \\
\hline \multirow[t]{2}{*}{ CA199, U/mL } & $>37$ & $-3.915 \pm 0.200$ & 0.336 \\
\hline & $<37$ & $-4.529 \pm 0.401$ & \\
\hline \multirow[t]{2}{*}{ Jaundice } & Yes & $-3.000 \pm 0.451$ & 0.006 \\
\hline & No & $-4.357 \pm 0.170$ & \\
\hline \multirow[t]{2}{*}{ Tumor size } & $<3 \mathrm{~cm}$ & $-3.751 \pm 0.198$ & 0.019 \\
\hline & $>3 \mathrm{~cm}$ & $-4.875 \pm 0.337$ & \\
\hline \multirow[t]{2}{*}{ Primary cancer site } & Head & $-4.204 \pm 0.252$ & 0.439 \\
\hline & Tail & $-3.920 \pm 0.256$ & \\
\hline \multirow[t]{3}{*}{ Tumor differentiation } & High & / & 0.721 \\
\hline & Moderate & $-4.013 \pm 0.337$ & \\
\hline & Low & $-4.056 \pm 0.215$ & \\
\hline \multirow[t]{2}{*}{ Metastasis } & Yes & $-3.929 \pm 0.3508$ & 0.9148 \\
\hline & No & $-4.087 \pm 0.2127$ & \\
\hline
\end{tabular}

wd-score weighted diagnosis score, CA199 carbohydrate antigen199 Note: $P$ values were calculated using $t$ test

\section{Discussion}

This study investigated the potential application of epigenetic markers in detecting $\mathrm{PDAC}$, revealing that the $5 \mathrm{mC}$ model, $5 \mathrm{hmC}$ model, and $5 \mathrm{mC}-5 \mathrm{hmC}$ combined model all showed high prediction accuracy.

Global DNA hypomethylation and a reduction in $5 \mathrm{hmC}$ levels are frequently observed in cancer $[29,30]$. Our study revealed the global depletion of $5 \mathrm{hmC}$ modifications in PDAC cfDNA samples, with the number of $5 \mathrm{hmC}$ modification sites decreasing as well. This finding was consistent with the results reported in other cancers, such as colorectal cancer, gastric cancer, and lung cancer $[20,23]$. However, global hypermethylation (Supplementary Figure 2C) instead of $5 \mathrm{mC}$ depletion was observed in the PDAC cfDNA samples. No significant difference in $5 \mathrm{mC}$ peak numbers between the PDAC samples and controls was observed, though the PDAC samples showed a higher median number. Similar results were reported in PDAC tissue [31], for example, the total number of $5 \mathrm{mC}$ peaks identified in pancreatic tumor tissues was larger than that in nontumor tissue samples, and more hypermethylated differentially methylated regions (DMRs) were observed in tumor tissues than in nontumor tissues. Likewise, in our study, the number of DMPs hypermethylated in PDAC was much larger than that in controls.

DNA modifications and histone modifications are two important types of epigenetic mechanisms that might be correlated with each other [30]. It has been reported that DNA modification along with histone modification have vital roles in chromosome architecture [32], and DNA methylation might serve as template for histone modification [27]. Increased $5 \mathrm{hmC}$ modification and depleted $5 \mathrm{mC}$ modification at the permissive histone modification sites in PDAC patients probably indicated elevated expression levels of genes regulated by the elements located in these regions. Deeper investigations combining RNA expression data may further reveal the interactive regulatory mechanism of various types of epigenetic modifications, comprising DNA modifications and histone modifications.

Previous studies focused on gene bodies and defined the DMRs with sliding windows by breaking the reference genome to screen candidate biomarkers [20, 22, 23]. Such methods might lead to statistically significant differences that are not biologically meaningful if few read counts are mapped to the regions in both the cases and controls. The $201 \mathrm{bp}$ fixed-width peak method would reduce the risks since more attention is paid to smaller regions with higher enrichment. Moreover, smaller regions are more convenient and cost-effective considering their further application in clinical practice.

We conducted an exploratory study combining $5 \mathrm{mC}$ and $5 \mathrm{hmC}$ profiling, and the results suggest that the integration of the two epigenetic approaches improve the diagnostic power as envisioned, particularly the prediction sensitivity in early-stage PDAC samples. The diagnostic effect of the combined model is better than that of the $5 \mathrm{hmC}$ model and $5 \mathrm{mC}$ model in our study as well as that of models reported by other groups [22, 33] (AUC 0.997 vs $0.94-0.96$ and 0.92). In addition, the prediction accuracy is much higher than that of the diagnostic method combining CA199 and Kras mutation (sensitivity 0.94 vs 0.78 ; specificity 0.95 vs 0.77 ) [34]. In our study, the CA199 positive patients (>37 U/L) were 80.3\% (Supplementary Table 5), lower than the diagnostic sensitivity of the prediction models.

In the clinic, digital evaluation criteria would be more preferred, so a wd-score was then computed according to the logistic model coefficients and modification level of the corresponding markers for each individual (Supplementary Table 5). The results demonstrated that the wd- 
scores of both the $5 \mathrm{mC}$ and $5 \mathrm{hmC}$ markers were higher in patients with jaundice. The possible reason may be that the methylation levels of the epigenetic markers are affected by jaundice, similar to the increase of serum CA199 levels in PDAC patients with jaundice. Nevertheless, the opposite situation is possible. In clinical practice, obstructive jaundice usually occurs when tumor oppresses the common bile duct if the tumor arises in the head of the pancreas. Three $5 \mathrm{hmC}$ markers involved in PI (phosphatidylinositol) metabolism was identified, including PIP5K1A, INPP4B, and PNPLA7. It was reported that the ratio of phosphatidylinositol was higher in the plasma of the children with obstructive jaundice than in normal [35]. Further study on non-cancer patients with jaundice are needed to analyze the problem. We speculated that the $5 \mathrm{hmC}$ markers identified in this study might be used for the early diagnosis of PDAC given that a significant difference in wd-scores between stage I patients and stage II to IV patients was observed.

Traditionally, age, tumor location, tumor size, tumor grade, TNM stage, and positive lymph node metastasis have been considered important prognostic factors. Although we have not obtained the survival data of the included participants, we compared the wd-scores (from the integrated model) with the aforementioned predictors in the PDAC group (Table 2). Interestingly, we found that patients with tumor size less than $3 \mathrm{~cm}$ had higher wd-scores, suggesting further exploration of the mechanism of early tumor progression through gene epigenetic modification.

Nevertheless, there are limitations in this study. For example, a model for early diagnosis was not able to be constructed due to the limited number of stage I patients $(n=$ 8). We are dedicated to collecting large-scale samples for further research. In the future, we will adopt this approach for the diagnosis of malignant and benign disease, especially precancerous lesions such as intraductal papillary mucinous neoplasms (IPMNs). The early diagnosis of PDAC is still the top priority of our subsequent work.

\section{Conclusions}

In summary, we have developed a robust noninvasive approach combining epigenetic biomarkers for detecting PDAC. Our study demonstrated that both $5 \mathrm{mC}$ and $5 \mathrm{hmC}$ biomarkers in cfDNA are effective in PDAC detection, and the $5 \mathrm{mC}-5 \mathrm{hmC}$ integrated model presents increased diagnostic power. Larger numbers of samples and more subtypes of pancreatic diseases are worthy of further investigation.

\section{Methods}

\section{Study design}

This study recruited 72 PDAC patients and 136 healthy controls to investigate the potential diagnostic value of
$5 \mathrm{mC}$ and $5 \mathrm{hmC}$ biomarkers in cfDNA for pancreatic cancer. PDAC was confirmed histopathologically. Healthy participants, enrolled from the community, had normal liver and renal functions, normal cardiopulmonary function, no history of cancer, and no viral infections. Participants who met the following conditions were excluded: chemotherapy or radiotherapy for malignant tumors, metastatic PDAC, or PDAC with other cancer.

For $5 \mathrm{hmC}$ profiling, 5 samples were excluded because of the poor quality or low quantity of the sample. Five samples did not have $5 \mathrm{mC}$ profiling data due to an insufficient amount of cfDNA. All of the 136 healthy control samples were applied to $5 \mathrm{hmC}$ sequencing and cfMeDIP-seq, but only 97 of them yielded $5 \mathrm{mC}$ data when the analysis started.

\section{Clinical sample collection and sample processing}

Blood was collected into BD Vacutainer ${ }^{\circ}$ EDTA tubes (Becton, Dickinson and Company, Cat\# 367525). Within $2 \mathrm{~h}$, plasma was separated from blood by centrifugation at $1600 \times g$ at $4 .{ }^{\circ} \mathrm{C}$ for $10 \mathrm{~min}$ followed by $16000 \times g$ at $4{ }^{\circ} \mathrm{C}$ for $10 \mathrm{~min}$. Then, cfDNA was extracted with a QIAseq cfDNA Extraction kit (a part of the QIAseq All-in-one Kit, Cat. No. 180025) and quantified by a Qubit fluorometer (Life Technologies). CfDNA (10-30 ng) was applied for library construction: adaptor ligation was facilitated using the QIAseq cfDNA Library Kit (a part of the QIAseq All-in-one Kit, Cat. No. 180025) following the protocol provided by the manufacturer, with spike-in controls added $(0.01 \mathrm{pg}$ of each amplicon per $10 \mathrm{ng}$ of cfDNA). The spike-in control, including 3 distinct lambda DNA amplification products ( 180 bp) (one without modification and the other two with $5 \mathrm{mC}$ and $5 \mathrm{hmC}$ modifications) were prepared following the method described by Chun-Xiao Song et al [23].

\section{Methylome profiling}

Ligated cfDNA (5-10 ng) was applied for methylome profiling following the previously published cfMeDIPseq protocol $[21,22]$ with minor modifications. We used the spike-in controls mentioned above to roughly assess the $5 \mathrm{mC}$ enrichment ratio instead of the methylated DNA (meDNA) and unmethylated DNA (unDNA) spike-in controls used in cfMeDIP-seq. In brief, cfDNA libraries with the spike-in control and the filler DNA, as well as Buffer A and Buffer B provided in the Magnetic Methylated DNA Immunoprecipitation Kit (Diagenode, C02010021), were incubated at $95^{\circ} \mathrm{C}$ for $10 \mathrm{~min}$ and immediately chilled on ice for $10 \mathrm{~min}$. Then, $75 \mu \mathrm{l}$ of incubation mix was incubated with anti-5mC antibody (Magnetic Methylated DNA Immunoprecipitation Kit, Diagenode, C02010021) on a rotating wheel at $4{ }^{\circ} \mathrm{C}$ for $17 \mathrm{~h}$, followed by purification with the Magnetic IPure 
kit v2 (Diagenode, Cat. No. C03010015). The final libraries were amplified with QIAGEN HiFi PCR Master Mix, $2 x$ and Primer Mix Illumina Libr. Amp (QIAseq All-in-one Kit, Cat. No. 180025) as follows: $98^{\circ} \mathrm{C}$ for $2 \mathrm{~min}$, followed by $9-12$ cycles of $98^{\circ} \mathrm{C}$ for $20 \mathrm{~s}, 60^{\circ} \mathrm{C}$ for $30 \mathrm{~s}, 72^{\circ} \mathrm{C}$ for 30 $\mathrm{s}$, and a final extension at $72{ }^{\circ} \mathrm{C}$ for $1 \mathrm{~min}$. Afterward, the amplified libraries were purified using $0.8 \times$ Beckman Agencourt AMPure XP beads (Cat. No. A63881) and quantified by a Qubit fluorometer (Life Technologies). Pair-end 150 bp sequencing was performed on the Illumina NovaSeq 6000 system by Novogene Co., Ltd. (Beijing).

\section{$5 \mathrm{hmC}$ sequencing}

$5 \mathrm{hmC}$ profiling was performed using the method reported previously-the cfDNA $5 \mathrm{hmC}$ sequencing method based on selective chemical labeling (hMe-Seal) [23]. All procedures followed the protocol described in the paper. In brief, cfDNA ligated with sequencing adaptors was incubated in a $25 \mu \mathrm{l}$ reaction solution containing HEPES buffer (50 mM, pH 8.0), $\mathrm{MgCl}_{2}$ (25 mM), $60 \mu \mathrm{M} \mathrm{N}$-UDP -Glc (Active Motif, Carlsbad, CA, USA), and $12.5 \mathrm{U} \beta$-glucosyltransferase (NEB) for $2 \mathrm{~h}$ at $37^{\circ} \mathrm{C}$.

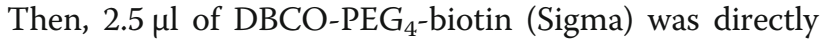
added and incubated for $2 \mathrm{~h}$ at $37^{\circ} \mathrm{C}$. Next, $10 \mu \mathrm{g}$ of sheared salmon sperm DNA (Life Technologies) was added. Subsequently, a Micro Bio-Spin 30 Column (Bio$\mathrm{Rad})$ was used to purify the DNA following the instructions, and the volume was adjusted to $25 \mu \mathrm{l}$. Afterward, purified DNA was incubated with $5 \mu \mathrm{l}$ of $\mathrm{C} 1$ streptavidin beads (Life Technologies, USA) in buffer 1 ( $5 \mathrm{mM}$ Tris pH 7.5, 0.5 mM EDTA, $1 \mathrm{M} \mathrm{NaCl}$, and 0.2\% Tween 20) for $30 \mathrm{~min}$. The beads were subsequently subjected to three 5-min washes each with buffer 1, buffer 2 (buffer 1 without $\mathrm{NaCl}$ ), buffer 3 (buffer 1 with $\mathrm{pH} 9$ ), and buffer 4 (buffer 3 without $\mathrm{NaCl}$ ). The beads were then resuspended in water and amplified with 9-12 cycles of PCR amplification (initial denaturing at $98^{\circ} \mathrm{C}$ for $45 \mathrm{~s}$, followed by $9-12$ cycles of denaturing at $98^{\circ} \mathrm{C}$ for $15 \mathrm{~s}$, annealing at $60^{\circ} \mathrm{C}$ for $30 \mathrm{~s}$, extension at $72^{\circ} \mathrm{C}$ for $30 \mathrm{~s}$, and a final extension at $72{ }^{\circ} \mathrm{C}$ for $5 \mathrm{~min}$ ). The amplified product was purified using AMPure XP beads. Pair-end $150 \mathrm{bp}$ sequencing was performed on the Illumina NovaSeq 6000 platform by Novogene Co., Ltd. (Beijing).

\section{Data processing}

For $5 \mathrm{hmC}$ and $5 \mathrm{mC}$ sequencing data alignment, the following methods were applied. The data quality of raw FASTQ files was checked by FastQC (version 0.11.8). The adapters of the raw FASTQ files were removed by Trimmomatic (version 0.38). Processed sequencing reads were aligned to hg19 and spike-in DNA using Bowtie2 (version 2.3.4.3) [36] with default parameters. The generated SAM files were filtered by SAMtools (version 1.9) [37] with the parameter settings of "-f 2 -F 1548 -q 30" to include high-quality, properly paired reads and then were converted to BAM format. Picard (version 2.18.23) (http://broadinstitute.github.io/picard) was employed to sort and index the filtered SAM files and to ensure the removal of duplicate reads before subsequent analysis. There were three types of spike-in DNA sequences, and capture efficiency, as a quality control measurement for $5 \mathrm{hmC}$ and $5 \mathrm{mC}$, was calculated as the counts of reads aligned to a type-specific spike-in DNA divided by the counts of reads aligned to the total spike-in DNA.

\section{Peak detection}

$5 \mathrm{hmC}$ and $5 \mathrm{mC}$ sequencing data peak detection was accomplished with the following steps. MACS2 (version 2.1.2) [38] was utilized to call peaks for each sequencing dataset. To obtain a high-confidence consensus peak list while considering differences in the sequencing depth and sample status (PDAC or healthy), a peak calling procedure was adopted as previously described [39]. In brief, the raw peak list within a single sample generated by MACS 2 was processed by first extending $100 \mathrm{bp}$ on either side of the peak summits, then normalizing MACS2 peak scores as "score per million" and finally removing overlapping peaks with an iterative removal procedure. Next, peaks defined as reproducible peaks with score per million $>=5$ were merged into group-specific (PDAC-specific or healthy-specific) peak lists by the same iterative procedure, and at last, the final consensus peak list was generated. Reproducible peaks are peaks emerging in at least N\% of cancer samples or healthy controls. Following the procedure mentioned above, nine consensus peak lists were generated with a set of values for $\mathrm{N}$ ( $\mathrm{N}$ range, $10 \sim 90$ with 10 increments). For each consensus peak list, we could obtain a zero-one matrix with rows representing consensus peaks and columns representing all samples, where one means a sample has a peak overlapping with a consensus peak and zero means no peak. Then, Fisher's exact test was applied to determine peaks with the most striking differences between PDAC samples and healthy controls. For $5 \mathrm{mC}$ data, $N=10$ resulted in the most significant peaks, and it was 30 in $5 \mathrm{hmC}$ data.

In particular, this peak list merging process was applied for $5 \mathrm{hmC}$ and $5 \mathrm{mC}$ data separately. All the peaks involved in the ENCODE hg19 blacklist [40], peaks that extend beyond any ends of chromosomes and peaks on chromosomes $\mathrm{X}, \mathrm{Y}$ or on the mitochondrial genome, were filtered.

Normalized pileup tracks generated by MACS2 were converted to bigwig format using bedGraphToBigWig from the UCSC Genome Browser and then put into the IGV for visualization $[25,26]$.

Bedtools (version 2.25.0) [41] was used to obtain the fragment counts of the final consensus peak list in each 
sample, and $5 \mathrm{hmC}$ or $5 \mathrm{mC}$ FPKM was then calculated. Differential peak analysis of the PDAC samples and healthy controls in the $5 \mathrm{hmC}$ and $5 \mathrm{mC}$ data was performed using Student's $t$ test (function $t$ test in $\mathrm{R}$ ), as well as differential analysis of PDAC samples between $5 \mathrm{mC}$ and $5 \mathrm{hmC}$ data. For the $t$-SNE plot, the FPKM of the consensus peak list was used as input, and the seed was set to 40 (perplexity was 52 ).

\section{Peak annotation}

To compare the distribution of peak numbers in PDAC and healthy samples for both $5 \mathrm{mC}$ and $5 \mathrm{hmC}$ data, the Wilcoxon test was used; the same was done for the comparison between $5 \mathrm{mC}$ and $5 \mathrm{hmC}$ data in PDAC samples. The genomic element distribution of peaks was determined by the percentage of peaks overlapping each element through BEDtools ( $>1 \mathrm{bp}$ ). The enrichment analysis of peak occupancy in genomic features was assessed by the odds ratio. Ngs.plot [42] was used to characterize metagene profiles. Gene Ontology (GO) analysis was performed by the ReactomePA R package (version 1.28.0) [43].

ChIP-Seq files of H3K4me1, H3K4me3, H3K36me3, H3K27ac, and H3K27me3 of the pancreatic cancer cell line PANC-1 provided by ENCODE were downloaded with the following identifiers: ENCFF520QXI, ENCF F213GUQ, ENCFF922RLL, ENCFF629BRY, and ENCF F915XVA [44]. BEDtools was adopted to obtain the percentage of peaks overlapping each histone modification region $(>1 \mathrm{bp})$.

\section{Biomarker selection and model construction}

The elastic-net regularization on a logistic linear regression model in the glmnet $\mathrm{R}$ package (version 2.0-18) [45] was chosen to establish prediction models. To filter more effective biomarkers for distinguishing PDAC samples from healthy controls, the following procedure was applied.

For the $5 \mathrm{mC}$ data set, we randomly split $70 \%$ of the healthy samples $(n=67)$ and $75 \%$ of the PDAC samples $(n=50)$ into the training set and the remaining samples into the validation set. To avoid overfitting, 5 rounds of 10-fold cross-validation was performed (Figure S8). The details were as follows: the training set was randomly divided into five folds, four of which were selected as the training subset, and the remaining one was the test subset. In each training subset, the DhMPs between PDAC samples and healthy controls with false discovery rate $($ FDR $)<0.01$ and $|\log 2 \mathrm{FC}|>0.8$ ( $t$ test) remained as candidates. Then, we performed 100 repeats to further select markers using the elastic-net model, and a panel of DhMPs in each training subset that appeared in at least $95 \%$ of the iterations was retained. Thus, 10-fold cross-validation was repeated 100 times each round.
Finally, the final markers observed in at least 3 rounds were used to build the final prediction model in the training set, and this model was utilized to predict the validation samples. The $\alpha$ was selected with maximize accuracy in the validation set over a grid of values from 0.1 to 0.9 .

For the $5 \mathrm{hmC}$ data set, $75 \%$ of the PDAC samples $(n=$ $39)$ and $75 \%$ of the healthy samples $(n=79)$ were randomly divided into the training set, and the remaining samples were placed into the validation set. The same procedure as that in the $5 \mathrm{mC}$ modeling pipeline was adopted, except for $\alpha$, which was 0.1 , and the criteria for the $t$ test, which was altered to $P$ value $<0.001$ and $|\log 2 \mathrm{FC}|>0.8$.

For the $5 \mathrm{mC}$ and $5 \mathrm{hmC}$ conjoint analysis, samples with both $5 \mathrm{mC}$ and $5 \mathrm{hmC}$ data were included. The training set consisted of $75 \%$ of the healthy samples $(n=$ 64 ) and $75 \%$ of the PDAC samples $(n=45)$, and the validation set consisted of the remaining data. The twentyfour $5 \mathrm{mC}$ biomarkers and the twenty-seven $5 \mathrm{hmC}$ biomarkers identified previously were combined for elasticnet model training (Figure S10).

The wd-score was calculated for each sample according to the biomarker model coefficients as follows:

$W d-$ score $=\operatorname{sum}(\operatorname{coef}(k) * F P K M(k))$, where $k$ represents the marker

\section{Statistical analysis}

Descriptive statistics of the characteristics of the participants were calculated using SPSS (v23.0, IBM, Armonk, NY, USA) as well as the chi-squared test for categorical variables. All tests were two-sided, and $P$ values $<0.05$ were considered statistically significant.

We also assessed the associations of the wd-score with prognostic indicators such as age over 60 , male sex, smoking status, alcohol consumption, CA199 over $37 \mathrm{U} /$ $\mathrm{ml}$, tumor size over $3 \mathrm{~cm}$, tumor location (head, body, and tail), tumor differentiation (high, moderate and low), and the occurrence of metastasis in PDAC patients using $t$ test to evaluate the application value of the $\mathrm{wd}$-score for the prognosis prediction of PDAC.

Statistical analyses were performed in $\mathrm{R}$ 3.6.3. The Wilcoxon-Mann-Whitney test was used to compare different groups except that ANOVA was applied to compare the wd-scores of $5 \mathrm{mC}, 5 \mathrm{hmC}$, and the integrated $5 \mathrm{mC}-5 \mathrm{hmC}$ model in different AJCC-staged pancreatic cancer patients. Raw $P$ values were corrected by Benjamini and Hochberg correction. The $\mathrm{R}$ packages RtSNE (version 0.15) [46] and pheatmap (version 1.0.12) were used for dimension reduction and clustering analysis. The glmnet package was utilized to construct prediction models. The roc function of the $\mathrm{R}$ package $\mathrm{pROC}$ (version 1.15.3) was used to generate receiver operating characteristic (ROC) curves and calculate the AUC. 


\section{Supplementary information}

Supplementary information accompanies this paper at https://doi.org/10. 1186/s13148-020-00898-2.

Additional file 1: Supplementary Figure 1. Percentage of reads mapped to the spike-in DNA. A. The $5 \mathrm{mC}$ spike-in DNA is specifically enriched in the $5 \mathrm{mC}$ libraries. B. The $5 \mathrm{hmC}$ spike-in DNA is specifically enriched in the $5 \mathrm{hmC}$ libraries. Error bars indicate Standard Deviation (SD). Supplementary Figure 2. Global change in $5 \mathrm{mC}$ and $5 \mathrm{hmC}$ level in PDAC. A. Boxplot of $5 \mathrm{mC}$ peak numbers from healthy controls and PDAC samples shows no significant difference. B. Boxplot of $5 \mathrm{hmC}$ peak numbers from healthy controls and PDAC samples shows significant larger numbers of peaks in PDAC samples. C. Genome browser view of the cell-free $5 \mathrm{mC}$ distribution in a $5 \mathrm{mb}$ region in chromosome 8. D. Genome browser view of the cell-free $5 \mathrm{hmC}$ distribution in a $3 \mathrm{mb}$ region in chromosome 7 . The overlapping tracks of healthy and PDAC are shown in line plot. ${ }^{*} P<0.05,{ }^{* *} P<0.01,{ }^{* * *} P<0.001,{ }^{* * *} P<1 e-5$, Wilcoxon test. PDAC, pancreatic ductal adenocarcinoma. Supplementary Figure 3. Genomic distribution of $5 \mathrm{mC}$ and $5 \mathrm{hmC}$ peaks. A. $5 \mathrm{mC}$ distribution in genomic features. B. Enrichment of $5 \mathrm{mC}$ peaks overlapping with distinct genomic elements. C. $5 \mathrm{hmC}$ peak distribution in genomic features. D. Enrichment of $5 \mathrm{hmC}$ peaks overlapping with distinct genomic elements. PDAC, pancreatic ductal adenocarcinoma; CDS, Coding DNA Sequence; 3 UTR, 3'untranslated region; 5'UTR, 5'untranslated region. Supplementary Figure 4. Comparison of the $5 \mathrm{mC}$ and $5 \mathrm{hmC}$ peaks. A. Venn diagram of overlap between $5 \mathrm{mC}$ and $5 \mathrm{hmC}$ peaks. B. Venn diagram of overlap between genes with $5 \mathrm{mC}$ modifications and genes with $5 \mathrm{hmC}$ modifications. Supplementary Figure 5. GO term enrichment analysis of specifically modified genes. A. 5mC-specific genes. B. 5hmC-specific genes. PDAC, pancreatic ductal adenocarcinoma. Supplementary Figure 6. Genome browser views of examples of specifically modified genes. A. ME1 gene in chromosome 6: 84,095-84,140 kb. B. PACRG gene in chromosome 6: 163,716-163,734 kb. C. FYN gene in chromosome 6: $112,132-112,148 \mathrm{~kb}$. D. RALB gene in chromosome 2: 121,000-121,030 kb. ME1, malic enzyme 1; PACRG, parkin coregulated; FYN, FYN protooncogene; $R A L B$, RAS like proto-oncogene B. Supplementary Figure 7. T-SNE analysis of $5 \mathrm{mC}$ FPKM. A. T-SNE plot of $5 \mathrm{mC}$ FPKM from PDAC and healthy samples in distinct batches after removing batch effect. B. T-SNE plot of $5 \mathrm{mC}$ FPKM from PDAC samples and healthy samples. PDAC, pancreatic ductal adenocarcinoma. Supplementary Figure 8. Flow chart of $5 \mathrm{mC}$ model construction. Supplementary Figure 9. T-SNE analysis of $5 \mathrm{hmC}$ FPKM. A. T-SNE plot of 5hmC FPKM from PDAC and healthy samples in distinct batches. B. T-SNE plot of 5hmC FPKM from PDAC and healthy samples. PDAC, pancreatic ductal adenocarcinoma. Supplementary Figure 10. Flow chart of $5 \mathrm{hmC}$ model construction. Supplementary Figure 11. Performance of the $5 \mathrm{hmC}$ model in distinguishing the subgroups of PDAC patients. A. Boxplot of the wd-scores in the resectable PDAC patients and unresectable PDAC patients. B. Boxplot of the wd-scores in PDAC patients with jaundice and those without jaundice. ${ }^{*} P$ $<0.05$, ${ }^{* *} P<0.01,{ }^{* * *} P<0.001$, ${ }^{* * *} P<1 e-5$, Wilcoxon test.

Additional file 2: Supplementary Table 1. Mapping summary of cfDNA $5 \mathrm{mC}$ sequencing data. Supplementary Table 2. Mapping summary of cfDNA $5 \mathrm{hmC}$ sequencing results. Supplementary Table $\mathbf{3}$. Differentially methylated peaks identified by $t$ test. Supplementary Table 4. List of $5 \mathrm{mC}$ markers used in model construction. Supplementary Table 5. Wd-scores of PDAC patients derived from distinct models. Supplementary Table 6. Differentially hydroxymethylated peaks identified by $t$ test. Supplementary Table 7. List of $5 \mathrm{hmC}$ markers used in model construction.

\section{Abbreviations}

5hmC: 5-Hydroxymethylcytosine; 5mC: 5-Methylcytosine; AJCC: American Joint Committee on Cancer; AUC: Area under the curve; cfDNA: Cell-free DNA; cfMeDIP-seq: Cell-free methylated DNA immunoprecipitation and highthroughput sequencing; ctDNA: Circulating tumor DNA; DhMPs: Differentially hydroxymethylated peaks; DMPs: Differentially methylated peaks; FPKM: Fragments per kilobase of gene per million mapped reads; hMeSeal: 5hmC-selective chemical labeling; IGV: Integrative Genomics Viewer; PDAC: Pancreatic ductal adenocarcinoma; $t$-SNE: t-distributed stochastic neighbor embedding analysis; wd-score: Weighted diagnosis score

\section{Acknowledgements}

The authors would like to acknowledge all the healthy and patient volunteers involved in this study. We thank all the recruiting and participating centers and technicians, TaiLai Biosciences for technical support and providing HPC Core, Qing Deng for sample collection and other members of Li and Xie labs for support and discussion.

\section{Authors' contributions}

JZ, AW, DX, and AL conceived the project. FC and JZ carried out the experiments. YH developed the methodology of data analysis with the help from $L X, K T$, and DX. YH and $X H$ completed the data processing and data analysis, and AW participated in the statistical analysis. AW, JY, ZG, and HL participated in the data acquisition. FC wrote the manuscript with the contributions of $\mathrm{YH}, \mathrm{XH}, \mathrm{AL}, \mathrm{JZ}$, and $\mathrm{DX}$. The authors read and approved the final manuscript.

\section{Funding}

This work was supported by grants: 31771426 from Chinese National Natural Science Foundation to Dan Xie; the expert funding of National Natural Science Foundation of China (81773174) to Ang Li; the 1.3 .5 project for disciplines of excellence-Clinical Research Incubation and Innovation Project, West China Hospital, Sichuan University (ZYJC18044) to Ang Li; and Science and technology project of Sichuan Province (20ZDYF2954) to Ailin Wei.

\section{Availability of data and materials}

https://pms.cd120.com/PDAC/index.html

\section{Ethics approval and consent to participate}

All subjects were recruited according to a West China Hospital Institutional Review Board-approved protocol, and informed consent was provided.

\section{Consent for publication}

All authors contributed to the manuscript and approve its submission.

\section{Competing interests}

The authors have declared that no competing interest exists.

\section{Author details}

${ }^{1}$ Frontier Science Center for Disease Molecular Network, State Key Laboratory of Biotherapy, West China Hospital, Sichuan University, Chengdu 610041, Sichuan Province, China. ${ }^{2}$ Department of Pancreatic Surgery, West China Hospital, Sichuan University, Chengdu 610041, Sichuan Province, China. ${ }^{3}$ Key Laboratory of Transplant Engineering and Immunology, Regenerative Medicine Research Center, West China Hospital, Sichuan University, Chengdu 610041, Sichuan Province, China.

Received: 3 May 2020 Accepted: 1 July 2020

Published online: 23 July 2020

\section{References}

1. Kamisawa T, Wood LD, Itoi T, Takaori K. Pancreatic cancer. The Lancet. 2016; 388(10039):73-85.

2. Crowley E, Di Nicolantonio F, Loupakis F, Bardelli A. Liquid biopsy: monitoring cancer-genetics in the blood. Nat Rev Clin Oncol. 2013;10(8): 472-84

3. Beaver JA, Jelovac D, Balukrishna S, Cochran R, Croessmann S, Zabransky DJ, et al. Detection of cancer DNA in plasma of patients with early-stage breast cancer. Clin Cancer Res. 2014;20(10):2643-50.

4. De Mattos-Arruda L, Weigelt B, Cortes J, Won HH, Ng CK, Nuciforo P, et al. Capturing intra-tumor genetic heterogeneity by de novo mutation profiling of circulating cell-free tumor DNA: a proof-of-principle. Annals of Oncology. 2014:25(9):1729-35.

5. Jiang P, Chan CW, Chan KC, Cheng SH, Wong J, Wong WW, et al. Lengthening and shortening of plasma DNA in hepatocellular carcinoma patients. Proc Natl Acad Sci U S A. 2015;112(11):E1317-25.

6. Rothe F, Laes JF, Lambrechts D, Smeets D, Vincent D, Maetens M, et al. Plasma circulating tumor DNA as an alternative to metastatic biopsies for mutational analysis in breast cancer. Ann Oncol. 2014;25(10):1959-65.

7. Liang W, Zhao Y, Huang W, Gao Y, Xu W, Tao J, et al. Non-invasive diagnosis of early-stage lung cancer using high-throughput targeted DNA 
methylation sequencing of circulating tumor DNA (ctDNA). Theranostics. 2019;9(7):2056-70.

8. Luo HY, Zhao Q, Wei W, Zheng LH, Yi SH, Li G, Wang WQ, Sheng H, Pu HY, Mo HY, Zuo ZX, et al. Circulating tumor DNA methylation profiles enable early diagnosis prognosis prediction and screening for colorectal cancer. Sci Transl Med. 2020;12(524):eaax7533.

9. Han J, Lv P, Yu JL, Wu YC, Zhu X, Hong LL, et al. Circulating methylated MINT2 promoter DNA is a potential poor prognostic factor in gastric cancer. Digest Dis Sci. 2014;59(6):1160-8.

10. Johnson KC, Houseman EA, King JE, von Herrmann KM, Fadul CE, Christensen BC. 5-Hydroxymethylcytosine localizes to enhancer elements and is associated with survival in glioblastoma patients. Nat Commun. 2016;7:13177.

11. Xu RH, Wei W, Krawczyk M, Wang W, Luo H, Flagg K, et al. Circulating tumour DNA methylation markers for diagnosis and prognosis of hepatocellular carcinoma. Nat Mat. 2017:16(11):1155-61.

12. Amatu A, Schirripa M, Tosi F, Lonardi S, Bencardino K, Bonazzina E, et al. High circulating methylated DNA Is a negative predictive and prognostic marker in metastatic colorectal cancer patients treated with regorafenib. Front Oncol. 2019:9.

13. Azad AA, Volik SV, Wyatt AW, Haegert A, Le Bihan S, Bell RH, et al. Androgen receptor gene aberrations in circulating cell-free DNA: biomarkers of therapeutic resistance in castration-resistant prostate cancer. Clin Cancer Res. 2015;21(10):2315-24.

14. Murtaza M, Dawson SJ, Tsui DW, Gale D, Forshew T, Piskorz AM, et al. Noninvasive analysis of acquired resistance to cancer therapy by sequencing of plasma DNA. Nature. 2013;497(7447):108-12.

15. Rakyan VK, Down TA, Balding DJ, Beck S. Epigenome-wide association studies for common human diseases. Nat Rev Genet. 2011;12(8):529-41.

16. Vakoc $C R$, Tuveson DA. Untangling the genetics from the epigenetics in pancreatic cancer metastasis. Nat Genet. 2017;49(3):323-4.

17. Chen YL, Hu CM, Hsu JT, Chang CC, Huang TY, Chiang PH, Chen WY, Chang YT, Chang MC, Tien YW, Lee EY. Cellular 5-hydroxylmethylcytosine content determines tumorigenic potential and prognosis of pancreatic ductal adenocarcinoma. Am J Cancer Res. 2018;8(12):2548-63.

18. Liu J, Jiang J, Mo J, Liu D, Cao D, Wang H, He Y, Wang H. Global DNA 5Hydroxymethylcytosine and 5-formylcytosine contents are decreased in the early stage of hepatocellular carcinoma. Hepatology. 2019;69(1):196-208.

19. Feinberg AP, Koldobskiy MA, Gondor A. Epigenetic modulators, modifiers and mediators in cancer aetiology and progression. Nat Rev Genet. 2016; 17(5):284-99.

20. Li W, Zhang X, Lu X, You L, Song Y, Luo Z, et al. 5-Hydroxymethylcytosine signatures in circulating cell-free DNA as diagnostic biomarkers for human cancers. Cell Res. 2017:27(10):1243-57.

21. Shen SY, Burgener JM, Bratman SV, De Carvalho DD. Preparation of cfMeDIP-seq libraries for methylome profiling of plasma cell-free DNA. Nat Prot. 2019;14(10):2749-80.

22. Shen SY, Singhania R, Fehringer G, Chakravarthy A, Roehrl MHA, Chadwick $D$, et al. Sensitive tumour detection and classification using plasma cell-free DNA methylomes. Nature. 2018;563(7732):579-83.

23. Song $C X$, Yin $S$, Ma L, Wheeler A, Chen $Y$, Zhang $Y$, et al. 5Hydroxymethylcytosine signatures in cell-free DNA provide information about tumor types and stages. Cell Res. 2017;27(10):1231-42.

24. Berdasco M, Esteller M. Clinical epigenetics: seizing opportunities for translation. Nat Rev Genet. 2019;20(2):109-27.

25. Robinson JT, Thorvaldsdottir H, Winckler W, Guttman M, Lander ES, Getz G, et al. Integrative genomics viewer. Nat Biotechnol. 2011;29(1):24-6.

26. Thorvaldsdottir H, Robinson JT, Mesirov JP. Integrative Genomics Viewer (IGV): high-performance genomics data visualization and exploration. Brief Bioinform. 2012;14(2):178-92.

27. Cedar $\mathrm{H}$, Bergman Y. Linking DNA methylation and histone modification: patterns and paradigms. Nat Rev Genet. 2009;10(5):295-304.

28. Consortium EP. An integrated encyclopedia of DNA elements in the human genome. Nature. 2012;489(7414):57-74.

29. Cai J, et al. Genome-wide mapping of 5-hydroxymethylcytosines in circulating cell-free DNA as a non-invasive approach for early detection of epatocellular carcinoma. Gut. 2019;68:2195-205.

30. Esteller M. Epigenetics in Cancer. N Engl J Med. 2008;358:1148-59.

31. Zhao Y, Sun J, Zhang H, Guo S, Gu J, Wang W, et al. High-frequency aberrantly methylated targets in pancreatic adenocarcinoma identified via global DNA methylation analysis using methylCap-seq. Clin Epigenet. 2014; 6(1):18.
32. Esteller M. Cancer epigenomics: DNA methylomes and histone-modification maps. Nat Rev Genet. 2007;8(4):286-98.

33. Collin F, Ning Y, Phillips T, McCarthy E, Scott A, Ellison C, et al. Detection of early stage pancreatic cancer using 5 -hydroxymethylcytosine signatures in circulating cell free DNA. BioRxiv. 2018.

34. Gu JN, Wang D, Huang Y, Lu Y, Peng CH. Diagnostic value of combining CA 19-9 and K-ras gene mutation in pancreatic carcinoma: a meta-analysis. Int J Clin Exp Med. 2014;7(10):3225-34..

35. Boon J, Broekhuyse RM, Van Munster P, Schretlen E. Abnormal pattern of the phospholipids of plasma and erythrocytes in four children with obstructive jaundice with abnormal spontaneous hemolysis. Clinica Chimica Acta. 1969;23:453-6.

36. Langmead B, Salzberg SL. Fast gapped-read alignment with Bowtie 2. Nat Methods. 2012;9(4):357-9.

37. Li H, Handsaker B, Wysoker A, Fennell T, Ruan J, Homer N, et al. The sequence alignment/map format and SAMtools. Bioinformatics. 2009;25(16): 2078-9.

38. Zhang Y, Liu T, Meyer CA, Eeckhoute J, Johnson DS, Bernstein BE, et al. Model-based analysis of ChIP-seq (MACS). Genome Biol. 2008:9(9):R137.

39. Corces MR, Granja JM, Shams S, Louie BH, Seoane JA, Zhou W, et al. The chromatin accessibility landscape of primary human cancers. Science. 2018; 362(6413):eaav1898.

40. Ewan BE. Summary for annotation file set ENCSR636HFF. https:/www. encodeproject.org/annotations/ENCSR636HFF/. Accessed 15 May 2008 - 31 Dec 2012.

41. Quinlan AR, Hall IM. BEDTools: a flexible suite of utilities for comparing genomic features. Bioinformatics. 2010;26(6):841-2.

42. Shen L, Shao N, Liu X, Nestler E. ngs.plot: Quick mining and visualization of next-generation sequencing data by integrating genomic databases. BMC genomics. 2014;15:284

43. Yu G, He Q-Y. ReactomePA: an R/bioconductor package for reactome pathway analysis and visualization. Mol BioSystems. 2016;12(2):477-9.

44. ENCODE ChIP-seq Experiment Matrix hg19 (2007 - 2012). Available from: https://www.encodeproject.org/. Accessed 21 Sept 2012 - 31 July 2016.

45. Friedman JHT, Tibshirani R. Regularization paths for generalized linear models via coordinate descent. J Stat Softw. 2010;33:1-22.

46. van der Maaten LHG. Visualizing data using t-SNE. Mach Learn Res. 2008;9: 2579-605.

\section{Publisher's Note}

Springer Nature remains neutral with regard to jurisdictional claims in published maps and institutional affiliations.
Ready to submit your research? Choose BMC and benefit from:
- fast, convenient online submission
- thorough peer review by experienced researchers in your field
- rapid publication on acceptance
- support for research data, including large and complex data types
- gold Open Access which fosters wider collaboration and increased citations
- maximum visibility for your research: over $100 \mathrm{M}$ website views per year
At BMC, research is always in progress.
Learn more biomedcentral.com/submissions 